International Journal of Nano Studies \& Technology (IJNST)

\title{
ISSN:2167-8685
}

\section{Synthesis of $\mathrm{Co}_{x} \mathrm{Ni}_{1-\mathrm{X}} \mathrm{Fe}_{2} \mathrm{o}_{4}(\mathrm{X}=\mathbf{0 . 0}, \mathbf{0 . 5}, 1.0)$ Nanoparticles by Chemical Co-Precipitation Route}

K. Maaz ${ }^{1 *}$, Ihsan A², Fatima I², Masood F², S. Karim¹.

Research Article

${ }^{1}$ Nanomaterials Research Group, Physics Division, PINSTECH, Islamabad, Pakistan.

${ }^{2}$ Department of Physics, COMSATS Institute of Information Technology, Islamabad, Pakistan.

\begin{abstract}
Magnetic nanoparticles $(\mathrm{NPs})$ of nickel-cobalt ferrite $\left(\mathrm{Co}_{\mathrm{x}} \mathrm{Ni}_{1-\mathrm{x}} \mathrm{Fe}_{2} \mathrm{O}_{4} ; \mathrm{x}=0.0,0.5,1.0\right)$ were synthesized by co-precipitation route. The
chemicals used were nickel chloride, cobalt chloride, ferric chloride, sodium hydroxide, and oleic acid. Sodium hydroxide was used as
the precipitating agent and oleic acid was used as the surfactant. $\mathrm{X}$-ray diffraction $(\mathrm{XRD})$ analysis confirmed the formation of single
phase nickel-cobalt ferrite nanoparticles in the size range $12-20 \mathrm{~nm}$. The obtained size of the nanoparticles is depending on the vari-
ation of different reaction parameters during the reaction and annealing time and temperature after the reaction. Hysteresis loops of
the samples confirmed the magnetic characteristics of the prepared $\left(\mathrm{Co}_{\mathrm{x}} \mathrm{Ni}_{1-\mathrm{x}} \mathrm{Fe}_{2} \mathrm{O}_{4} ; \mathrm{x}=0.0,0.5,1.0\right)$ nanoparticles.
Key Words: Ferrite Nanoparticles; Magnetic Properties; Hysteresis Loop
\end{abstract}
*Corresponding Author:

K. Maaz,

Nanomaterials Research Group, Physics Division,

PINSTECH, Islamabad, Pakistan.

E-mail: maaz@impcas.ac.cn

Received: August 09, 2014

Accepted: August 23, 2014

Published: August 25, 2014

Citation: K. Maaz, Ihsan A, Fatima I, Masood F, S. Karim. (2014). Synthesis of $\mathrm{Co}_{\mathrm{x}} \mathrm{Ni}_{1-\mathrm{X}} \mathrm{Fe}_{2} \mathrm{O}_{4}(\mathrm{X}=0.0,0.5,1.0)$ Nanoparticles by Chemical Co-Precipitation Route, Int J Nano Stud Technol, 03(04), 55-58. doi: http://dx.doi.org/10.19070/2167-8685-1400011

Copyright: K. Maaz (C) 2014 This is an open-access article distributed under the terms of the Creative Commons Attribution License, which permits unrestricted use, distribution and reproduction in any medium, provided the original author and source are credited.

\section{Introduction}

In recent years, nanotechnology occupied a great area of interest for the scientists working in the field of nanoscience and nanotechnology. This is due to the reason that various properties of the materials change as the size approaches to the nano-scale. At this scale the chemical and physical properties of the materials are entirely differ from their bulk counterparts that result to alter their optical, mechanical, chemical and magnetic properties [1-4]. Magnetic nanoparticles are more focused by the researchers as they have wide range of applications in medical, data storage, optical filters and military services [5-7]. Ferrite is the class of magnetic materials that exhibit strong magnetic properties. Magnetic nature of the nanoparticles is determined by many factors including their chemical composition, shape, size, and inter-particle interactions in these materials. Ferrites are the combination of various metal oxides with one of the constituent as the iron oxide among them. They possess high electrical resistivity and high magnetic permeability. Ferrites possess spinel (cubic) structure with chemical formula as MFe2O4; where $\mathrm{M}$ represents the divalent metal ion such as $\mathrm{Zn}^{2+}, \mathrm{Fe}^{2+}, \mathrm{Mg}^{2+}, \mathrm{Mn}^{2+}, \mathrm{Cd}^{2+}$ and $\mathrm{Fe}$ in (+3) oxidation state. In single ferrite molecule there are eight divalent metal ions, 16 ferric $\left(\mathrm{Fe}^{+3}\right)$ ions and 32 oxygen ions. Oxygen atoms form face centered cubic structure in ferrite molecule. In nickel- and cobalt-ferrite cations $\left(\mathrm{M}^{+2}\right)$ occupy octahedral and tetrahedral sites with main structure made by oxygen atoms. In ferrites each tetrahedral site is surrounded by four oxygen atoms while each octahedral site is surrounded by six oxygen atoms.

Several methods have been used for synthesis of nanoparticles that include sol-gel, hydrothermal, micro-emulsion and co-precipitation technique [8-14]. In our case we have used coprecipitation method to prepared nickel-cobalt ferrite nanoparticles [15]. The advantage of this method over the others is that one can easily overcome the problem of controlling size and size distribution of the nanoparticles. Coprecipitation method is a smooth and convenient way which involves conversion of metal chlorides into the metal hydro-oxides. These hydro-oxides are then heated up to $80^{\circ} \mathrm{C}$ for formation of ferrites nanoparticles. At this stage the prepared nanoparticles are amorphous and need further heat treatment (i.e. annealing) at $600^{\circ} \mathrm{C}$ to get pure crystalline form of the nanoparticles. In our case oleic acid was used as the surfactant to avoid agglomeration of the smaller nanoparticles to form the bigger particles.

The structural characterization was performed with X-ray diffraction (XRD) while vibrating sample magnetometer (VSM) was used for magnetic analysis of the samples. XRD involves the identification of crystalline phases in the prepared nanoparticles. Debey Scherrer formula was used to calculate the size of the particles by using the strongest peak in XRD pattern.VSM measurements were performed under an applied field of $10 \mathrm{kOe}$. In this paper we present synthesis, structural, and magnetic characterization of nickel ferrite, cobalt ferrite, and nickel-cobalt- nanoparticles with sizes 12,16 , and $20 \mathrm{~nm}$.

\section{Synthesis Procedure}

For synthesis of nanoparticles coprecipitation route was followed [15]. The chemicals used were cobalt chloride, nickel chloride, ferric chloride, sodium hydroxide and oleic acid. Double distilled deionized water was used a solvent in order avoid any impurities in the final nanoparticles. A 0.4 molar aqueous (M) solution of iron chloride was mixed with $0.2 \mathrm{M}$ aqueous solution of cobalt- 
and nickel-chloride under vigorous magnetic stirrerring. $3 \mathrm{M}$ solution of sodium hydroxide was added drop-wise to the mixture solution, which causes precipitation in the mixture. Few drops of oleic acid (2-3) were added to the mixture, which act as the surfactant and coating material on the nanoparticles. The mixture was heated at $80^{\circ} \mathrm{C}$ for 1 hour to get the actual reaction started. After the reaction the mixture was cooled to room temperature in order to settle down the NP's precipitates at the bottom of the beaker. The mixture was washed with double distilled water 2-3 times in order to remove the water dissolvable impurities and excess oleic acid from the nanoparticles. The mixture was centrifuged@7000 rpm for $5 \mathrm{~min}$ in order to isolate the precipitates from the supernatant liquid. The final product was heated at $70^{\circ} \mathrm{C}$ in the electric oven to dry the nanoparticles. The acquired substance was then grinded in to the powder form to get the nanoparticles. At this stage powder nanoparticles were in amorphous form. Finally, the sample was annealed at $600^{\circ} \mathrm{C}$ for $6 \mathrm{hrs}$ to get the desired nanoparticles in crystalline form.

\section{Results and Discussion}

X-diffraction analysis of the samples synthesized by chemical coprecipitation route is shown in Figure.1-3. The XRD analyses of the samples annealed at $600^{\circ} \mathrm{C}$ confirm that the final product is cubic spinel structure of nickel-cobalt ferrite $\left(\mathrm{CO}_{\mathrm{x}} \mathrm{Ni}_{1-\mathrm{x}} \mathrm{Fe}_{2} \mathrm{O}_{4}\right.$; $\mathrm{x}$ $=0.0,0.5,1.0)$ nanoparticles. All the peaks in the XRD pattern were found to correspond with cubic spinel structure of $\mathrm{NiFe}_{2} \mathrm{O}_{4}$ and $\mathrm{CoFe}_{2} \mathrm{O}_{4}$ according to the standard JCPDS cards (742081 for $\mathrm{NiFe}_{2} \mathrm{O}_{4}$ and 791744 for $\mathrm{CoFe}_{2} \mathrm{O}_{4}$ ). In all cases the peaks were found at $30,35.4,37.2,43,53.6,57$, and $62.5^{\circ}$. These peaks were indexed according to the JCPDS cards mentioned above. The corresponding plans to these peaks were found to be (220), (311), (222), (400), (422), (511), and (440). The X-ray diffraction patterns confirm the crystalline nature of the obtained nanoparticles. The particle size was calculated by Debye Scherrer's equation[16]:

$$
\text { Prticle size }=\frac{\overline{\mathrm{K} \lambda}}{B \cos \theta_{B}}
$$

Where $\mathrm{K}$ is the shape constant, $\lambda$ is the wavelength of the $\mathrm{x}$ rays used, and $\mathrm{B}$ is the full width half maximum (FWHM) of the strongest peak in the XRD. Thus using this formula, the particle sizes were found to be 12,16, and $20 \mathrm{~nm}$ for $\mathrm{NiFe}_{2} \mathrm{O}_{4}$, $\mathrm{Ni}_{0.5} \mathrm{Co}_{0.5} \mathrm{Fe}_{2} \mathrm{O}_{4}$, and $\mathrm{CoFe}_{2} \mathrm{O}_{4}$ respectively. In our previous work, the size of the particles was observed to be increasing linearly with annealing temperature of the samples [15]. It has been reported that the annealing process generally decreases the lattice defects and strains; however it can also cause coalescence of smaller grains that results in the increasing average grain size of the nanoparticles [17]. The observed increase in particle size with annealing temperature is most likely due to the fact that higher annealing temperature and time enhances the coalescence process, which results in the increasing grain size of the samples thereby increasing the particle size of the nanoparticles.

Magnetic characterization of the nanoparticles was performed by vibrating sample magnetometer (VSM) at room temperature (300 $\mathrm{K})$, with maximum applied field upto $10 \mathrm{kOe}$. Figure. 4, 5, and 6 show the $\mathrm{M}(\mathrm{H})$ loops of the samples taken at room temperature. The coercivity and saturation magnetization (Ms) at room temperature as depicted from the $\mathrm{M}(\mathrm{H})$ loops were 1210,550 , and 184 Oe for cobalt ferrite, nickel-cobalt ferrite and nickel ferrite respectively. While the saturation magnetization values obtained from these loops were found to be 63,55 , and $41 \mathrm{emu} / \mathrm{g}$. The coercivity values obtained in this case were found to be larger than the corresponding bulk values of the samples while the Ms values were found to be smaller that their bulk values at room temperature. The relatively large coercivity values are consistent with the pronounced growth of magnetic anisotropy inhibiting the alignment of the moment along applied field direction [15]. While the smaller saturation magnetization as compared to their bulk values may be explained below.

The smaller MS values for nanoparticles as compared to the bulk values are attributed to the pronounced surface effects in case of the nanoparticles. The surface is considered to be composed of canted or disordered spins that prevents the core spins to align along the field direction, resultantly decreasing the saturation magnetization of the nanoparticles. Sometimes the surface becomes magnetically dead or inert that has negligible magnetization and causing reduced saturation magnetization of the nanoparticles [15].

Figure. 1. X-ray diffraction pattern of $\mathrm{CoFe}_{2} \mathrm{O}_{4}$ nanoparticles prepared by coprecipitation method, after annealing at $600^{\circ} \mathrm{C}$ for 6 hrs.

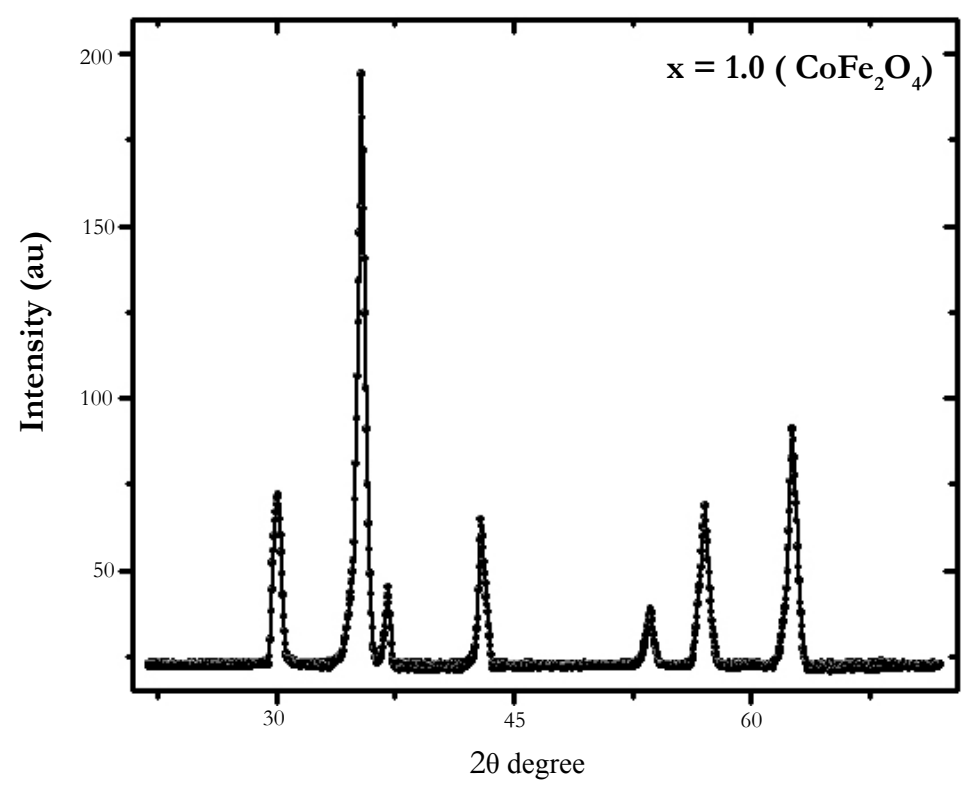


Figure. 2. XRD pattern of $\mathrm{Ni}_{0.5} \mathrm{Co}_{0.5} \mathrm{Fe}_{2} \mathrm{O}_{4}$ nanoparticles synthesized by coprecipitation route annealed at $600^{\circ} \mathrm{C}$ for 6 hrs.

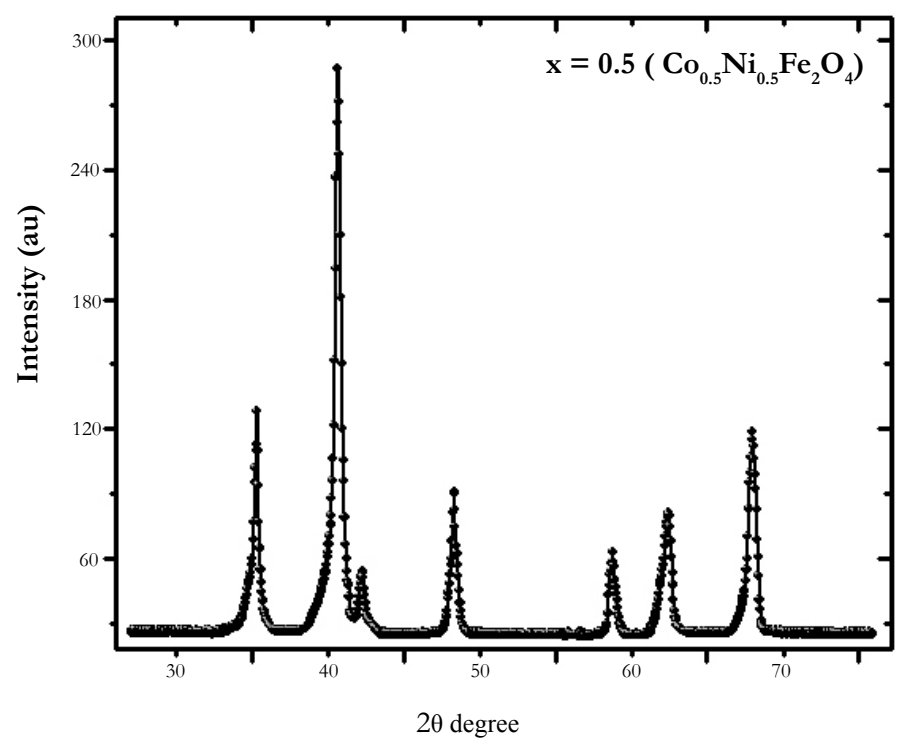

Figure. 3.X-ray diffraction pattern of $\mathrm{NiFe}_{2} \mathrm{O}_{4}$ nanoparticles prepared by coprecipitation method with average size of 12

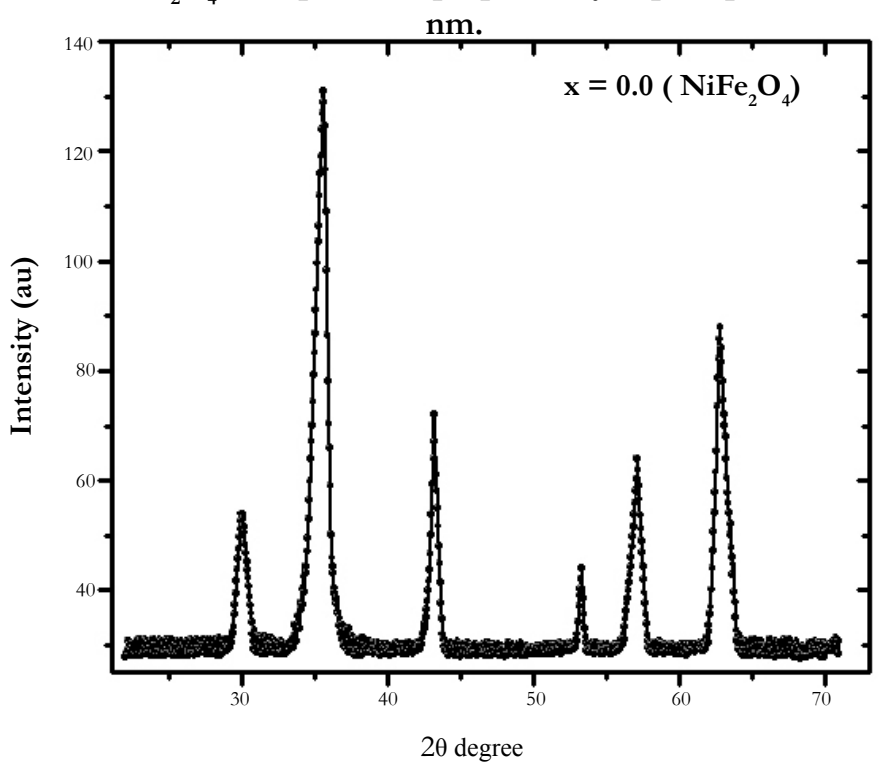

Figure. 4. Hysteresis loop of $20 \mathrm{~nm} \mathrm{CoFe}{ }_{2} \mathrm{O}_{4}$ nanoparticles at room temperature $(300 \mathrm{~K})$ at maximum applied field of 10

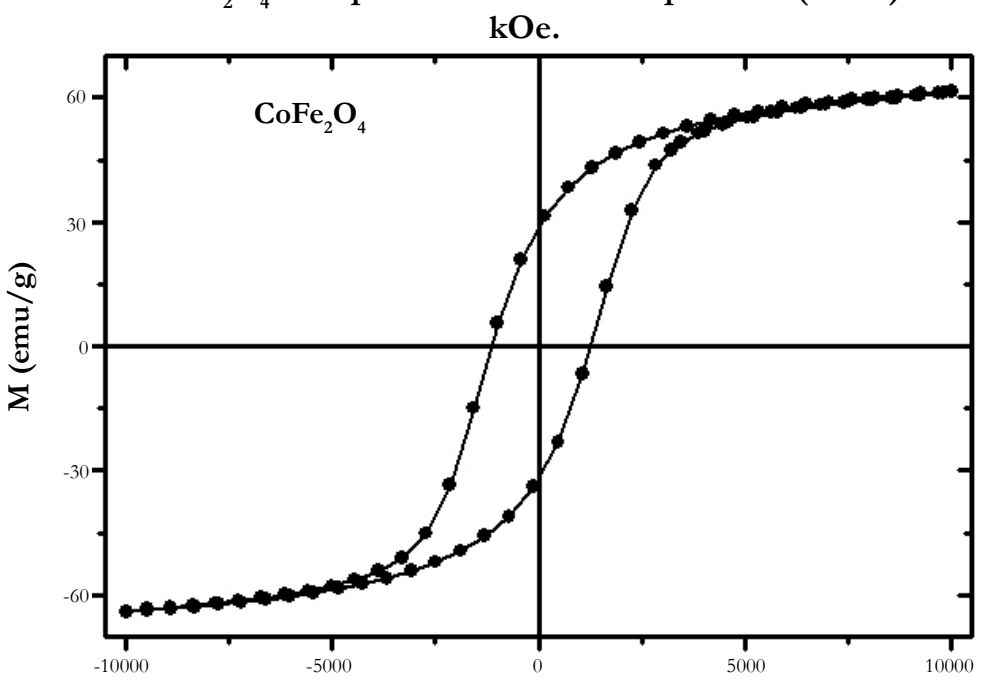

Field (Oe)

\section{Conclusion}

Magnetic nanoparticles (NPs) of nickel-cobalt ferrite $\left(\mathrm{Co}_{\mathrm{x}} \mathrm{Ni}_{1}\right.$ $\left.{ }_{x} \mathrm{Fe}_{2} \mathrm{O}_{4} ; \mathrm{x}=0.0,0.5,1.0\right)$ were successfully synthesized by co- precipitation route. X-ray diffraction (XRD) analysis confirmed the formation of single phase nickel-cobalt ferrite nanoparticles in the size range 12-20 nm calculated by using Debye Scherrer's equation. The coercivity of the obtained nanoparticles was found to be larger than the bulk values while the saturation magnetiza- 


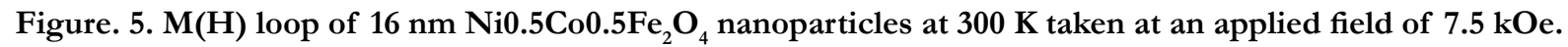

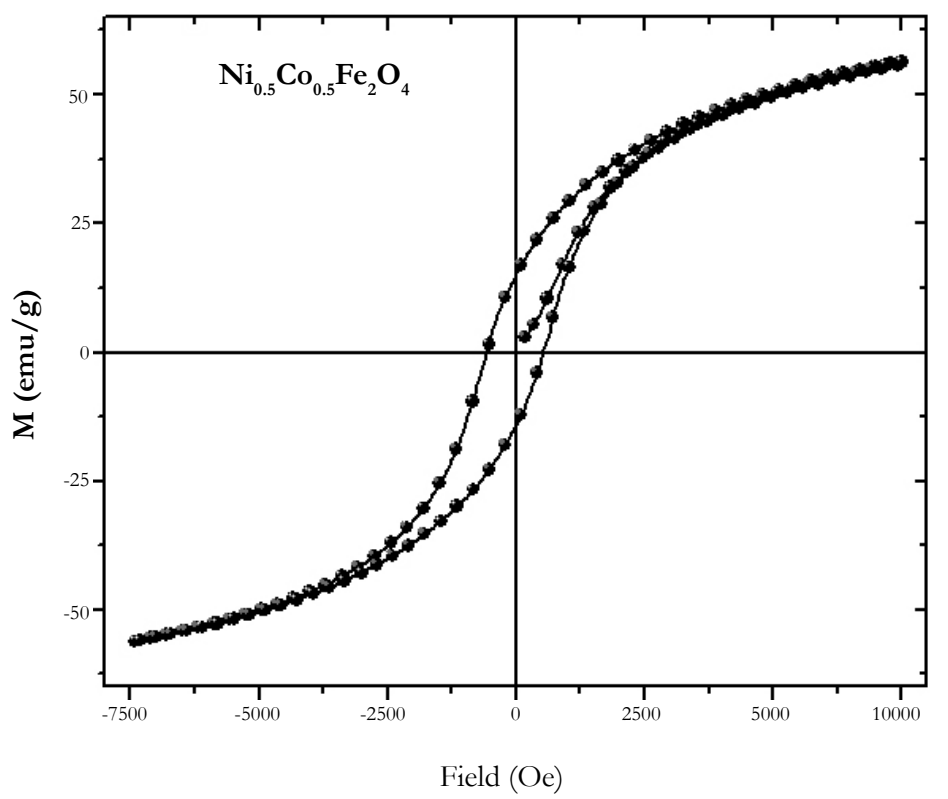

Figure. 6. $\mathrm{M}(\mathrm{H})$ loop of $12 \mathrm{~nm} \mathrm{NiFe} \mathrm{O}_{4}$ nanoparticles at $300 \mathrm{~K}$ at an applied field of $5 \mathrm{kOe}$.

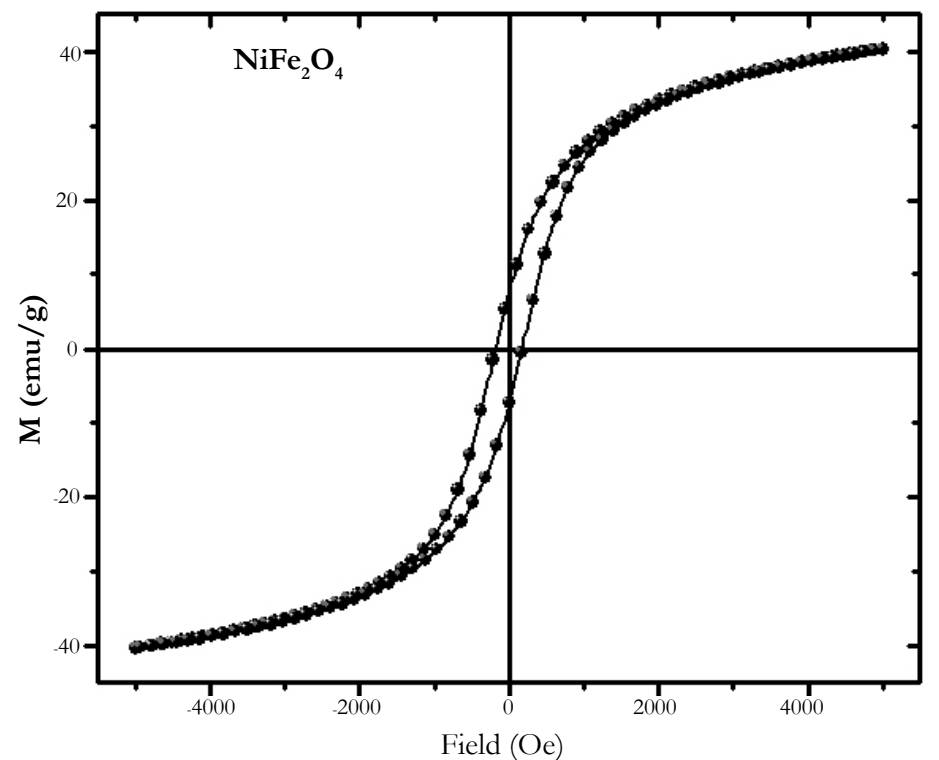

tion was found smaller that their bulk values at room temperature. The large coercivity values were attributed to the pronounced growth of magnetic anisotropy while the smaller saturation magnetization was attributed to the pronounced surface effects in case of nanoparticles.

\section{Acknowledgment}

The authors are thankful to Javed Bashir, Rubina Shaheen, Syed Z.Hussain for their cooperation in XRD analysis of the samples. While the central diagnostic laboratory (CDL), PINSTECH is also acknowledged.

\section{References}

[1]. M. Dariel, L.H. Bennett, D.S. Lashmore, P. Lubitz, M. Rubinstein, et al (1987) Properties of Electro-deposited Co-Cu Multilayers J. Appl. Phys. 61:4067.

[2]. W.D. Williams, N. Giordano (1986) Experimental study of localization and electron- electron interaction effects in thin Au wires Phys. Rev. B 33: 8146.

[3]. T.M. Whitney, J.S. Jiang, P.C. Searson, C.L. Chien, (1993) Science 261:1316.

[4]. L. Piraux, J.M. George, J.F. Despres, C. Leroy, E. Ferain, et al (1994). Giant magnetoresistance in magnetic multilayered nanowires Appl. Phys. Lett. 65 :2484.
[5]. P. Tartaj, M.D. Morales, V.V. Sabino, (2003) The preparation of magnetic nanoparticles for applications in biomedicine,J. Phys. D 36: R-182.

[6]. S.D. Bader (2006) Colloquium: Opportunities in nanomagnetism Rev. Mod. Phys. 78:1.

[7]. C.A. Ross (2001) Ann. Rev. Mater. Res. 31:203

[8]. D.H. Chen, Y.Y. Chen, J. Colloid Interface Sci. 9 (2001) 235.

[9]. J.J. Kingsley, K. Suresh, K.C. Patil, J. Mater. Sci. 25 (1990) 1305.

[10]. D.H. Chen, X.R. He (2001) Synthesis of Nickel Ferrite Nanoparticles by Sol-Gel Method Bull. Mater. Res. 36:1369.

[11]. S.Z. Zhang, G.L. Messing, J. Am. Ceram. Soc. 73 (1990) 61.

[12]. D.H. Chen, Y.Y. Chen, J. Colloid Interface Sci. 41 (2001) 236.

[13]. J.L. Duan, J. Liu, H.J. Yao, Dan Mo, M.D. Hou, et al (2008). Controlled synthesis and diameter-dependent optical properties of $\mathrm{Cu}$ nanowire arrays;Mater. Sci. Eng. B 147:57.

[14]. D. Mo, J. Liu, H.J. Yao, J.L. Duan, M.D. Hou, et al (2008) Preparation and characterization of $\mathrm{CdS}$ nanotubes and nanowires by electrochemical synthesis in ion-track templates, J. Cryst. Growth 310:612.

[15]. K. Maaz, A. Mumtaz, S.K. Hasanain, A. Ceylan, (2007) Journal of Magnetism and Magnetic Materials, J. Magn. Magn. Mater. 308:289-295.

[16]. T. Fukui, C.Sakura, M.Okuyama, (1992) Preparation of $\mathrm{Pb}(\mathrm{Zr} 1-\mathrm{xTix}) \mathrm{O} 3$ powders from complex alkoxide and their lower-temperature crystallization J. Mater Res. 7 :791; S. Chkoundali, S. Ammar, N. Jouini, F. Fievet, P. Molinie, M. Danot, F. Villain, J-M. Greneche, J. Phys.: Condens. Matter 16 (2004) 4357-4372.

[17]. T.P. Raming, A.J.A. Winnubst, C.M. van Kats, P. Philipse, (2002)The synthesis and Magnetic properties of nanosized hematite $(\alpha-\mathrm{Fe} 2 \mathrm{O} 3)$ particles ,J. Colloid Interface Sci. 249:346 